\title{
Supporting Information for Methods
}

\section{Disease Activity Index (DAI)}

The colitis disease activity index (DAI) was evaluated based on the following parameters: (a) body weight, (b) stool consistency and (c) rectal bleeding. Each score was determined as follows: body weight loss (0: none, $1: 1-5 \%, 2: 5-10 \%, 3: 10-15 \%, 4:>15 \%)$, stool consistency (0: normal, 1 and 2: loose stool, 3 and 4: diarrhea) and rectal bleeding (0: absent, 1: blood-tinged, 2 and 3: slight bleeding, 4: gross bleeding). The DAI was the average of the scores across the three-above parameters. 
Table S1. The contents of individual phenolic compounds of LPP powder determined by HPLC-DAD.

\begin{tabular}{lllll}
\hline compound & & standard curve & correlation coefficient & content $(\mathrm{mg} / \mathrm{g})$ \\
\hline \multirow{3}{*}{ phenolic acids } & gallic acid & $\mathrm{y}=35.599 \mathrm{x}-23.621$ & 0.9996 & $0.76 \pm 0.05$ \\
& vanillic acid & $\mathrm{y}=30.925 \mathrm{x}-23.026$ & 0.9999 & $0.33 \pm 0.03$ \\
& caffeic acid & $\mathrm{y}=49.386 \mathrm{x}-38.765$ & 0.9998 & $0.25 \pm 0.04$ \\
& ferulic acid & $\mathrm{y}=59.155 \mathrm{x}-35.806$ & 0.9999 & $3.72 \pm 0.21$ \\
& syringic acid & $\mathrm{y}=44.469 \mathrm{x}-50.828$ & 0.9999 & $0.45 \pm 0.06$ \\
\hline \multirow{3}{*}{ flavonoids } & (+)-catechin & $\mathrm{y}=13.293 \mathrm{x}-38.438$ & 0.9991 & $2.06 \pm 0.24$ \\
& procyanidin B2 & $\mathrm{y}=2.9985 \mathrm{x}-5.9016$ & 0.9989 & $2.01 \pm 0.11$ \\
& quercetin-3- $O$-rutinose-7- $O-\alpha-\mathrm{L}-$ & $\mathrm{y}=4.2939 \mathrm{x}-14.683$ & 0.9999 & $223.79 \pm 16.67$ \\
& rhamnoside & & & $82.02 \pm 0.31$ \\
& procyanidin A2 & $\mathrm{y}=2.0113 \mathrm{x}-9.2104$ & 0.9998 & $25.32 \pm 1.70$ \\
\hline
\end{tabular}


Table S2. The component of standard rat chow diet.

\begin{tabular}{llll}
\hline ingredient & & content $(\mathrm{g})$ & kcal \\
\hline protein & casein, 30 mesh & 200 & 800 \\
& L-cystine & 3 & 12 \\
carbohydrate & corn starch & 397 & 1590 \\
& maltodextrin & 132 & 528 \\
& sucrose & 100 & 400 \\
& cellulose & 50 & 0 \\
fat & soybean oil & 70 & 630 \\
& t-butylhydroquinone & 0.5 & 0 \\
mineral mix S10022G & & 35 & 0 \\
vitamin mix V10037 & & 10 & 40 \\
choline bitartrate & & 2.5 & 0 \\
\hline total & & 1000 & 4000 \\
\hline
\end{tabular}


Table S3. The rules of histological scoring.

\begin{tabular}{|c|c|c|c|c|}
\hline score & inflammation & crypt damage & ulceration & edema \\
\hline 0 & no infiltrate & none & none & none \\
\hline 1 & $\begin{array}{l}\text { few cells limited to } \\
\text { submucosa }\end{array}$ & $\begin{array}{l}\text { some crypt damage, } \\
\text { spaces between crypts }\end{array}$ & $\begin{array}{l}\text { little, focal } \\
\text { ulcers }\end{array}$ & present \\
\hline 2 & $\begin{array}{c}\text { obvious presence of } \\
\text { inflammatory cells in } \\
\text { submucosa, limited to focal } \\
\text { areas }\end{array}$ & $\begin{array}{l}\text { larger spaces between } \\
\text { crypts, loss of goblet } \\
\text { cells, some shortening } \\
\text { of crypts }\end{array}$ & $\begin{array}{l}\text { frequent small } \\
\text { ulcers }\end{array}$ & \\
\hline 3 & $\begin{array}{c}\text { infiltrate present in both } \\
\text { submucosa and lamina } \\
\text { propria, limited to focal areas }\end{array}$ & $\begin{array}{l}\text { large areas without } \\
\text { crypts, surrounded by } \\
\text { normal crypts }\end{array}$ & $\begin{array}{l}\text { large areas } \\
\text { lacking surface } \\
\text { epithelium }\end{array}$ & \\
\hline 4 & $\begin{array}{l}\text { large amount of infiltrate in } \\
\text { submucosa, lamina propria } \\
\text { and surrounding blood } \\
\text { vessels, covering large areas } \\
\text { of mucosa }\end{array}$ & no crypts & & \\
\hline 5 & $\begin{array}{l}\text { transmural inflammation } \\
\text { (mucosa to muscularis) }\end{array}$ & & & \\
\hline
\end{tabular}


Table S4. Primer sequences used for RT-qPCR in colonic tissue.

\begin{tabular}{|c|c|}
\hline gene & primer sequence $\left(5^{\prime}-3^{\prime}\right)$ \\
\hline \multirow[t]{2}{*}{ TNF- $\alpha$} & F: AGCCGATGGGTTGTACCTTG \\
\hline & R: ATAGCAAATCGGCTGACGGT \\
\hline \multirow[t]{2}{*}{ IL-6 } & F: CACAGAAGGAGTGGCTAAGGA \\
\hline & R: GCACTAGGTTTGCCGAGTAGAT \\
\hline \multirow[t]{2}{*}{ IL-1 $\beta$} & F: GGGCTGGACTGTTTCTAATGC \\
\hline & R: CTTGTGACCCTGAGCGACC \\
\hline \multirow[t]{2}{*}{ IFN- $\gamma$} & F: AACAACCCACAGGTCCAGC \\
\hline & R: CAGCAGCGACTCCTTTTCC \\
\hline \multirow[t]{2}{*}{ iNOS } & F: CGTTCCTGGAGGTGCTTGA \\
\hline & R: TCTCGGGTGCGGTAGGTG \\
\hline \multirow[t]{2}{*}{ COX-2 } & F: CTGCCAATAGAACTTCCAATCC \\
\hline & R: CCATAGAATAATCCTGGTCGGT \\
\hline \multirow[t]{2}{*}{ MCP-1 } & F: GTGCTGACCCCAAGAAGGA \\
\hline & R: TGAGGTGGTTGTGGAAAAGG \\
\hline \multirow[t]{2}{*}{ TLR-4 } & F: CGCTCTGGCATCATCTTCA \\
\hline & R: TTTTCCATCCAATAGGGCAT \\
\hline \multirow[t]{2}{*}{$\mathrm{ZO}-1$} & F: CTTGGGGAGGGAGGGTCA \\
\hline & R: GGCATTCCTGCTGGTTACATG \\
\hline \multirow[t]{2}{*}{ Occludin } & F: TGCGGTGACTTCTCCAAACT \\
\hline & R: GGGGAACGTGGCCGATAT \\
\hline \multirow[t]{2}{*}{ Claudin-1 } & F: CCAAGAGCCCCTATGTCACTC \\
\hline & R: TGCTTAGCAACAGAAGTTCCAG \\
\hline \multirow[t]{2}{*}{ FFAR2 } & F: TTGTTCTACTTCTCCTCCTCCG \\
\hline & R: CCCCTGGCTGTAGGTTGC \\
\hline \multirow[t]{2}{*}{ FFAR3 } & F: GGCTGGTCTGGTCAGTGTAGTC \\
\hline & R: CCCCTGGCTGTAGGTTGC \\
\hline \multirow[t]{2}{*}{ NLRP3 } & F: AGCCCCATGAGTTCCCTTAA \\
\hline & R: TCCCAGTGCCCAGTCCAA \\
\hline \multirow[t]{2}{*}{ GAPDH } & F: AAGAAGGTGGTGAAGCAGG \\
\hline & R: GAAGGTGGAAGAGTGGGAGT \\
\hline
\end{tabular}




\section{Supporting Information for Results}

Figure S1. Rarefaction curve of OTU number of feces from different groups.

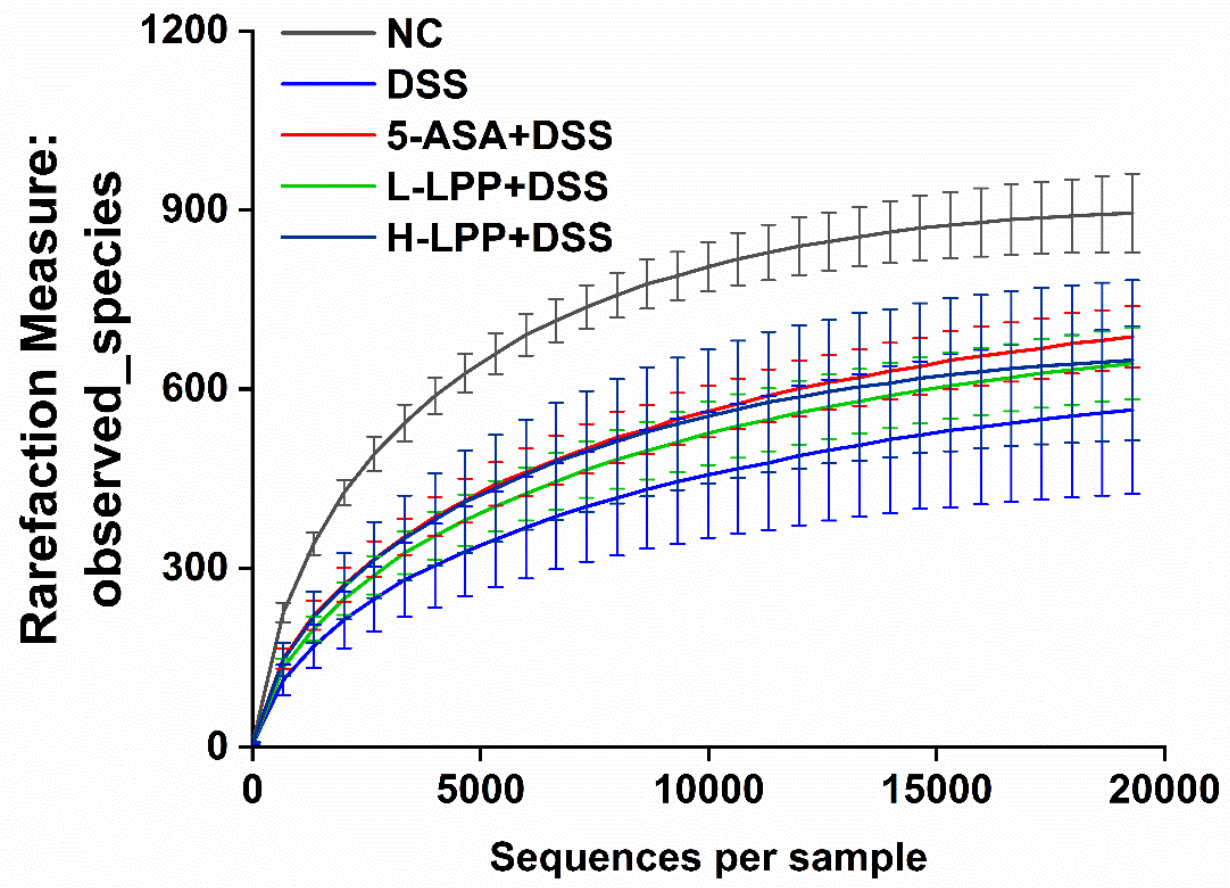


Figure S2. Effects of LPP supplementation on the compositions of Bacteroides at the species level. Bars with no letter in common are significantly different $(p<0.05)$.
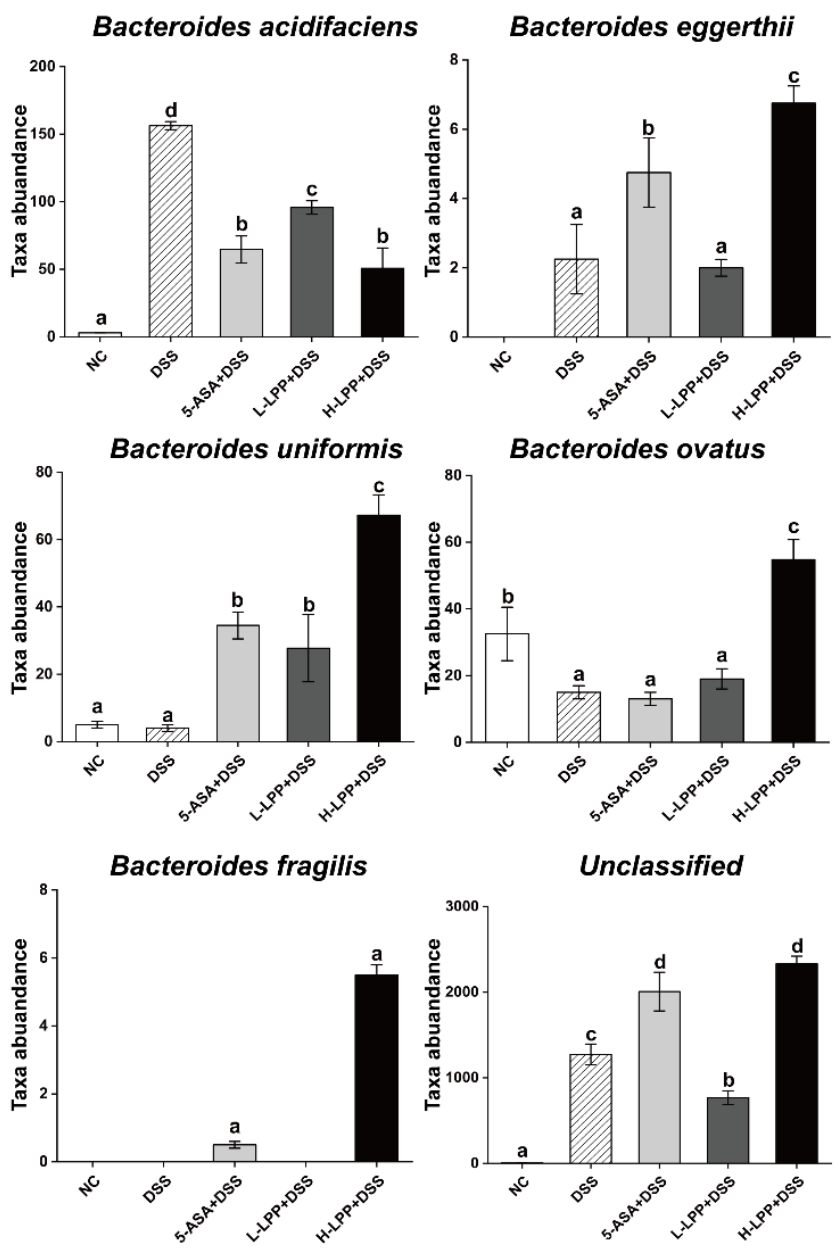